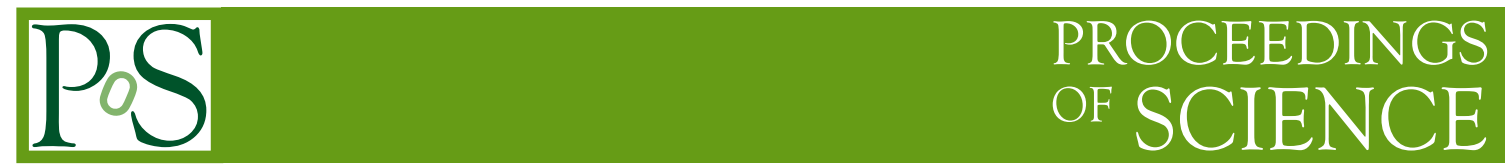

\title{
CMS Results in Electroweak Physics
}

\author{
Ilaria Segoni for the CMS Collaboration* \\ CERN \\ E-mail: ilaria.segoni@cern.ch
}

Electroweak results from CMS are presented based on $2.9 \mathrm{pb}^{-1}$ of pp collision data taken at $7 \mathrm{TeV}$. Precise measurements of inclusive cross sections establish standard model benchmark processes. Measurements of differential cross sections and lepton-based asymmetries test event generators and constrain parton distribution functions. The production of jets in association with a weak vector boson is studied in detail, leading to tests of perturbative QCD calculations as well as models of the underlying event.

Workshop on Discovery Physics at the LHC -Kruger 2010

December 05-10, 2010

Kruger National Park, Mpumalanga, South Africa

${ }^{*}$ Speaker. 


\section{1. $W$ and $Z$ inclusive cross section measurement}

The study of $\mathrm{W}$ and $\mathrm{Z}$ production allows for tests of perturbative $\mathrm{QCD}$ and parton distribution functions (PDF). Measurements at lower center of mass energy at $\mathrm{S} \bar{p} p \mathrm{~S}[1,2]$ and Tevatron [3, $4,5]$ are in good agreement with theoretical expectations. We report on new measurements at significantly higher energies using proton proton collision data at $\sqrt{s}=7 \mathrm{TeV}$ recorded with the CMS detector [6] using $2.9 \mathrm{pb}^{-1}$ of data [7]. The updated results using the full $2010 \mathrm{CMS}$ dataset, corresponding to $36 \mathrm{pb}^{-1}$ of data, are reported in [8] and [9].

Vector bosons are reconstructed from the decays into electron and muon channels. Electrons are required to be in the fiducial volume of the electromagnetic calorimeter (ECAL), i.e. $|\eta|<2.5$, with $1.4442<|\eta|<1.566$ excluded, to have passed the High Level Trigger (HLT) requirement of $p_{T}>15 \mathrm{GeV}$ and the offline requirement of $p_{T}>20 \mathrm{GeV}$. The electron reconstruction algorithm accounts for energy loss due to bremsshtrahlung. To reduce contamination from jets faking electrons, electron candidates are further identified based on the spatial matching between the ECAL supercluster and the track in the $\eta$ and $\phi$ coordinates, the supercluster shower spread along the $\eta$ direction and the energy leakage in the hadron calorimeter (HCAL). Muon candidates are required to have $|\eta|<2.1$, to have passed the HLT requirement of $p_{T}>9 \mathrm{GeV}$ and the offline requirement of $p_{T}>20 \mathrm{GeV}$. Additional quality requirements are applied in order to retain only candidates with a good $p_{T}$ determination. Both electrons and muons are required to be isolated from other tracks and energy deposits in the calorimeters in order to reduce contamination from decays in flight and, especially for electron channels, jets faking leptons.

Due to the undetected neutrino in the final state, $W$ events are characterized by a large "missing transverse energy" $\left(\mathbb{E}_{T}\right)$, i.e. the unbalanced transverse energy in the event. The reconstruction algorithm utilizes measurements from all detectors (tracking systems, muon chambers, calorimeters) in order to achieve superior resolution and accuracy on the $\mathbb{E}_{T}$ measurement. $W$ signal event yields are determined from an unbinned maximum likelihood fit to the $\mathbb{E}_{T}$ for the electron channel and from a templated fit to the $\mathrm{W}$ transverse mass $\left(M_{T}\right)$ in the muon channel. $M_{T}$ is defined as $M_{T}=\sqrt{\left.2 p_{T} \times M E T \times(1-\cos \Delta \phi)\right)}$ where $\Delta \phi$ is the angle in the $x y$-plane between the lepton $p_{T}$ and the $\mathbb{E}_{T}$ direction. Signal and EWK backgrounds shapes are taken from simulation using POWHEG[10]. The signal shape is corrected event-by-event by correction factors derived from a study of the hadronic recoil distributions in $Z \rightarrow l l$ events. The dominant background contamination derives from multi-jet events. For the $W(\mu v)$ signal extraction, this background component is modeled in the fit with a template derived from a data control sample where the muon isolation requirement is inverted. The $M_{T}$ distribution is distorted due to correlations between $\mathscr{E}_{T}$ and the lepton isolation. Also in this case, corrections are applied to remove this distortion based on a study of the hadronic recoil in $Z \rightarrow \ell^{+} \ell^{-}$events. For the $W(e v)$ signal extraction multi-jet events are described with an analytical function determined on a data control sample with reversed electron identification requirement on the spatial matching between Tracker and ECAL.

The di-lepton invariant mass $M_{l l}$ is used for the $Z$ signal extraction. In the electron case we utilize orthogonal methods, based on simulation and data, to determine the number of background events in the region $60 \mathrm{GeV}<M_{l l}<120 \mathrm{GeV}$. For the muon channel an unbinned maximum likelihood fit to the $M_{l l}$ distribution is performed.

The final $W$ and $Z$ cross sections are determined from the formula $\sigma \times B=N_{S i g} /(A \times \varepsilon \times L)$ 

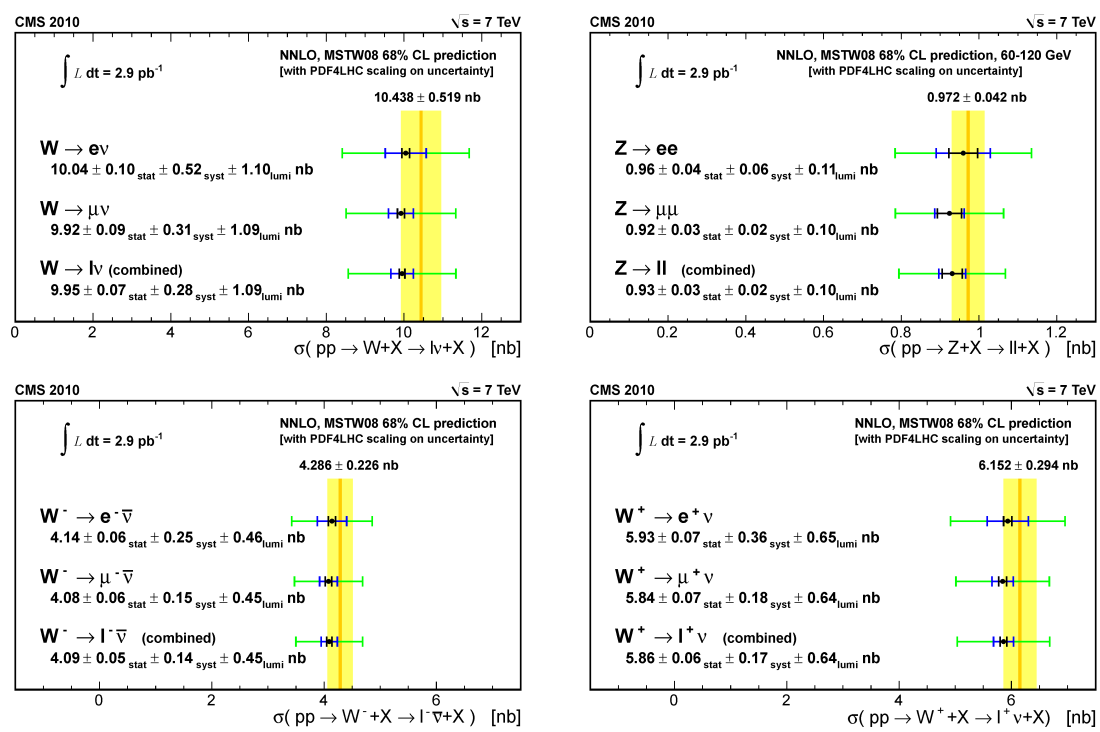

Figure 1: Summary of the production cross sections for $\mathrm{W}$ and $\mathrm{Z}$ (top). Bottom row show separately the cross section results for $W^{-}$(left) and $W^{+}$(right).

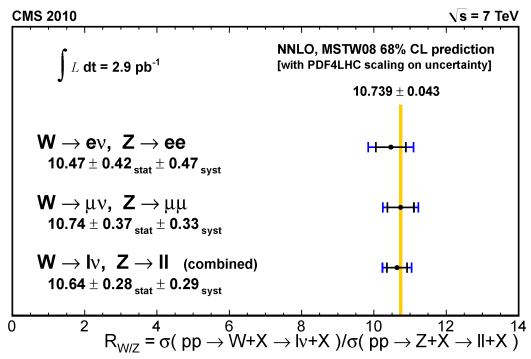

Figure 2: Summary of the $W / Z$ cross section ratio.

where $B$ is the branching ratio of the reconstructed decay mode, $N_{S i g}$ the number of observed signal events, $A$ the acceptance, $\varepsilon$ the signal selection efficiency and $L$ the integrated luminosity. The acceptance is evaluated with simulation, and it is affected by theoretical uncertainties. For this reason results are quoted both before and after correcting for the acceptance. The efficiency is based on data driven correction factors applied to the simulation efficiency determination. The correction factor are derived with a tag and probe method on $Z$ (11) samples. By applying such corrections we remove possible bias due to imperfections in the simulation. The dominant source of systematic uncertainty (11\%) comes from the estimation of the integrated luminosity. The second contribution to the final uncertainty is the statistical uncertainty on the efficiency correction factors (up to 5.9\% for the $Z$ (ee) channel). Other source of uncertainty are smalller and originate from leptons and $\mathbb{E}_{T}$ scale and resolution, and from the background subtraction.

The cross-section results are reported in Figures 1,2 and 3. We find everywhere consistency between the different channels and good agreement with theoretical calculations. With an experimental uncertainty that is lower than the uncertainties on theoretical prediction, the vector boson cross section measurements can already be used for improving the uncertainty on the luminosity 

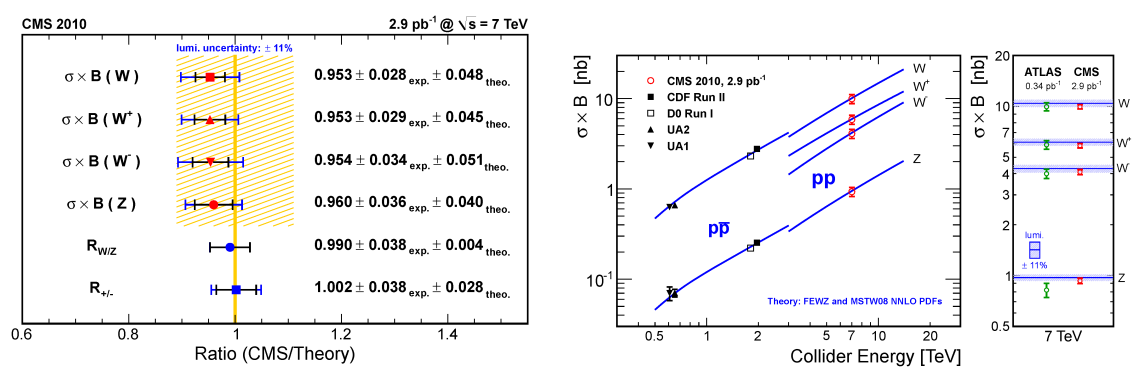

Figure 3: Left: summary of the ratios of the CMS measurement compared to the theoretical expectations (shaded area indicates the $11 \%$ luminosity uncertainty). Right: summary of $W$ and $Z$ cross section measurements for CMS and other experiments. The lines are NNLO theory predictions.

determination.

\section{Studies of differential cross sections}

Several studies of differential vector boson production cross sections are ongoing, including the rate of producing jets in association with $\mathrm{W}$ bosons, the $W$ charge asymmetry, the backwardforward asymmetry in $Z$ decays. The production of vector bosons with jets constitutes an important background in searches for new physics phenomena and for studies of the top quark, a precise measurement of the cross section and an understanding of the jet and lepton kinematics is essential in order to improve the sensitivity for searches. Preliminary studies of $W(l v)+$ jets based on the first $198 n b^{-1}$ of data are shown in Figure 4, for the leading jet $p_{T}$, and in Figure 5, for the associated jet rates, using a jet threshold of $30 \mathrm{GeV}$. Data are compared to predictions based on MADGRAPH [11] matrix element calculations plus PYTHIA [12] parton shower and to predictions based on parton PYTHIA only. Different tunes for the underlying event modeling [13] are used for the matrix element calculators. The predictions are normalized to NNLO predictions of the cross sections [14]. The data are in very good agreement with calculations based on matrix element, better than with the predictions from PYTHIA, especially as the number of jets increases.

\section{References}

[1] C. Albajar et al. [UA1 Collaboration], Z. Phys. C 44, 15 (1989).

[2] J. Alitti et al. [UA2 Collaboration], Z. Phys. C 47, 11 (1990).

[3] D. Acosta et al. [CDF II Collaboration], Phys. Rev. Lett. 94, 091803 (2005) [arXiv:hep-ex/0406078].

[4] A. A. Affolder et al. [CDF Collaboration], Phys. Rev. Lett. 84, 845 (2000) [arXiv:hep-ex/0001021].

[5] B. Abbott et al. [D0 Collaboration], Phys. Rev. D 61, 072001 (2000) [arXiv:hep-ex/9906025].

[6] R. Adolphi et al. [CMS Collaboration], JINST 3, S08004 (2008).

[7] V. Khachatryan et al. [CMS Collaboration], JHEP 1101, 080 (2011) [arXiv:1012.2466 [hep-ex]].

[8] CMS Collaboration, CMS PAS EWK-10-005 (2011), available from http://cdsweb.cern.ch/record/1337017?ln=en 


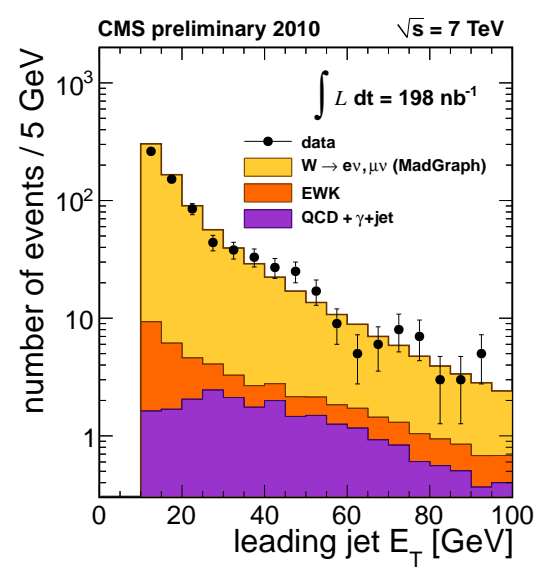

Figure 4: Leading jet $p_{T}$ spectrum in $W(l v)+$ jets events (at least one jet with $p_{T}>15 \mathrm{GeV}$. Data are compared to MADGRAPHplus PYTHIA predictions normalized to the NLO cross section.
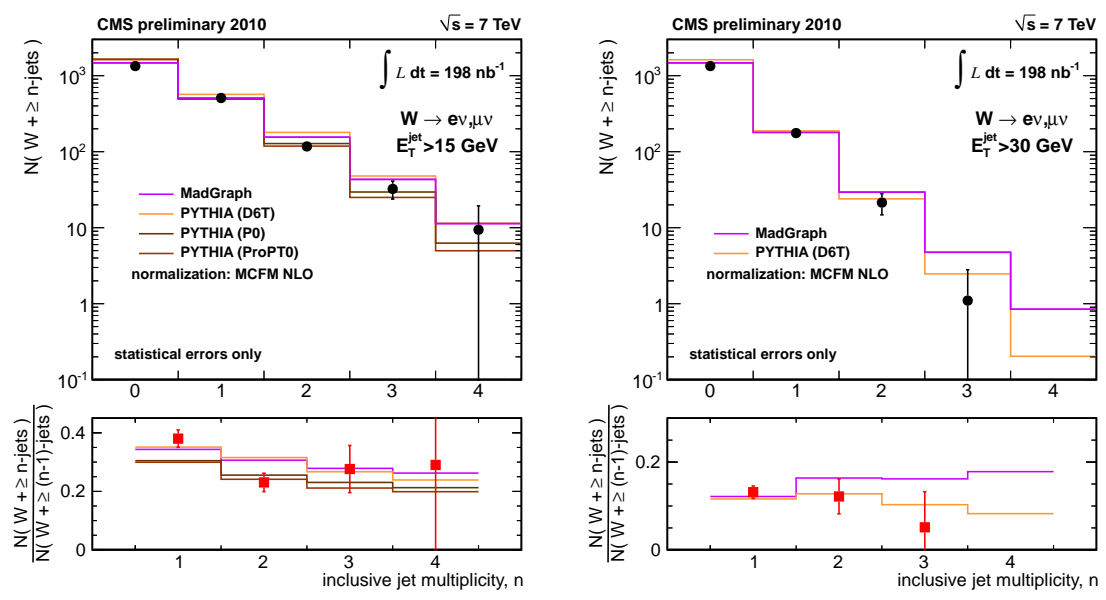

Figure 5: Rates of jets produced in association with a $W$ for a jet $p_{T}$ threshold of $15 \mathrm{GeV}$ (left) and $30 \mathrm{GeV}$ (right). Data are compared to several theoretical predictions and tend to favor the calculation based on matrix element.

[9] CMS Collaboration, CMS PAS EWK-10-012 (2011), available from http://cdsweb.cern.ch/record/1337018?ln=en

[10] S. Alioli, P. Nason, C. Oleari and E. Re, JHEP 0807, 060 (2008) [arXiv:0805.4802 [hep-ph]].

[11] F. Maltoni and T. Stelzer, JHEP 0302, 027 (2003) [arXiv:hep-ph/0208156].

[12] T. Sjostrand, S. Mrenna and P. Z. Skands, JHEP 0605, 026 (2006) [arXiv:hep-ph/0603175].

[13] P. Z. Skands, Phys. Rev. D 82, 074018 (2010) [arXiv:1005.3457 [hep-ph]].

[14] K. Melnikov and F. Petriello, Phys. Rev. D 74, 114017 (2006) [arXiv:hep-ph/0609070]. 